\title{
Chemical effects during the formation of various types of femtosecond laser-generated surface structures on titanium alloy
}

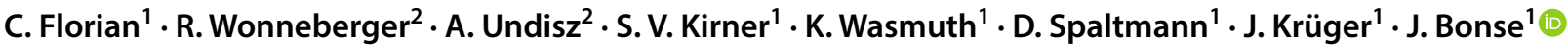

Received: 6 January 2020 / Accepted: 26 February 2020 / Published online: 12 March 2020

(c) The Author(s) 2020

\begin{abstract}
In this contribution, chemical, structural, and mechanical alterations in various types of femtosecond laser-generated surface structures, i.e., laser-induced periodic surface structures (LIPSS, ripples), Grooves, and Spikes on titanium alloy, are characterized by various surface analytical techniques, including X-ray diffraction and glow-discharge optical emission spectroscopy. The formation of oxide layers of the different laser-based structures inherently influences the friction and wear performance as demonstrated in oil-lubricated reciprocating sliding tribological tests (RSTTs) along with subsequent elemental mapping by energy-dispersive X-ray analysis. It is revealed that the fs-laser scan processing $(790 \mathrm{~nm}, 30 \mathrm{fs}, 1 \mathrm{kHz})$ of near-wavelength-sized LIPSS leads to the formation of a graded oxide layer extending a few hundreds of nanometers into depth, consisting mainly of amorphous oxides. Other superficial fs-laser-generated structures such as periodic Grooves and irregular Spikes produced at higher fluences and effective number of pulses per unit area present even thicker graded oxide layers that are also suitable for friction reduction and wear resistance. Ultimately, these femtosecond laser-induced nanostructured surface layers efficiently prevent a direct metal-to-metal contact in the RSTT and may act as an anchor layer for specific wear-reducing additives contained in the used engine oil.
\end{abstract}

Keywords Laser-induced oxide layer · Laser-induced periodic surface structures · LIPSS · Femtosecond laser processing · Tribology $\cdot$ Surface chemistry $\cdot$ XRD $\cdot$ GD-OES

\section{Introduction}

The fabrication of laser-induced periodic surface structures (LIPSS) on metals, semiconductors, and dielectrics has been experimentally demonstrated when irradiated with linearly polarized high-intensity ultrashort laser pulses. The formation process is influenced by the materials' optical, thermal, and mechanical properties [1-4]. Depending on the specific final application, other factors may be relevant such as the chemistry of the irradiated areas, the resulting crystallinity, and its final optical

C. Florian

camilo.florian-baron@bam.de

$\triangle$ J. Bonse

joern.bonse@bam.de

1 Bundesanstalt für Materialforschung und -prüfung (BAM), Unter den Eichen 87, 12205 Berlin, Germany

2 Otto Schott Institute of Materials Research (OSIM), Chair of Metallic Materials, Friedrich-Schiller-Universität Jena, 07743 Jena, Germany response [5]. In recent years, such periodic surface structures have been fabricated systematically for applications in optics, medicine, fluid transport, wetting, and tribology [5-10], exploring different laser processing parameters (fluence, wavelength, repetition rate, polarization, angle of incidence, number of pulses per spot) [5, 11-19], in different atmospheres (air, vacuum, reactive gasses) [20-23]. The simplest case of LIPSS is represented by parallel lines that are formed at the sample surface usually perpendicular to the laser polarization with periods that can be 100 times smaller than the laser beam diameter. In this way, it is possible to generate structures with periods even below the optical diffraction limit following a simple one-step fabrication process. Their periods are typically used to classify the laser-generated structures as low spatial frequency LIPSS (LSFL), when $\Lambda_{\mathrm{LSFL}} \sim \lambda$, or as high spatial frequency LIPSS (HSFL) for $\Lambda_{\mathrm{HSFL}}<\lambda / 2$ [5]. When the laser fluence and the effective number of pulses per spot increase from the conditions used for the formation of regular LIPSS, other topographic structures emerge that are known as micrometric Grooves and Spikes [24, 25]. The 
Grooves morphology corresponds to quasiperiodic surface structures that are usually parallel to the laser polarization featuring periods of a few micrometers, whereas the Spikes morphology consists of irregularly arranged blunt or sharp conical surface structures with average distances and heights of up to a few tens of micrometers [24, 25]. Upon scan processing, the Grooves and Spikes may be superimposed by LIPSS (generated in the low fluence wing of the Gaussian beam distribution at local fluences close to the ablation threshold), forming jointly together a hierarchical heterogeneous surface structure. Such hierarchical micro-nanostructure can feature applications for controlling the wettability of surfaces [9, 26-28], or for reducing the cell growth [7] in medical applications, e.g., on miniaturized leadless pacemakers that are implanted directly into the heart [29].

LSFL are the most desired type of structures for many applications, due to the simplicity to produce them on different materials and the rather moderate overall fabrication time required. Besides, these structures can be analyzed by different surface analytical techniques including optical and chemical surface characterization. Due to the rough morphology of Grooves and Spikes structures, they are often difficult to characterize. Given their low reflectivity due to the intrinsic high roughness, optical characterization often finds its limits when trying to obtain directly reflected light from such structures. Electron-based chemical analyses such as energy-dispersive X-ray analysis (EDX), Auger electron spectroscopy (AES), or X-ray photoelectron spectroscopy (XPS) may reveal the presence of specific chemical elements at those high roughness structures; however, these techniques generally suffer from constraints regarding the analytical information from both the depth and the topographic artifacts, which finally limits the quantification of elements and their actual distribution in depths, particularly for EDX [30, 31], where the information depths typically range between some hundreds of nanometers and a few micrometers [30]. Electron beam-based techniques with small information depths of the order of a few nanometers such as AES or XPS were successfully combined with ion sputtering to establish a depth profiling, but at suitable sputter conditions (few $\mathrm{kV}$ acceleration voltages) that approach is usually limited to total removal depths in the sub-micrometer range in reasonable analytic acquisition times.

The potentially most powerful but most time-consuming analytical method is transmission electron microscopy (TEM), where in the first step a cross-sectional lamella of the region of interest at the surface has to be prepared by deposition of a protecting capping layer followed by focused ion beam etching. In the second step, after subsequent milling of the lamella to electron beam transparency, direct electron beam-based imaging or diffraction can be applied to visualize the subsurface region, for identifying chemical elements or to reveal crystalline or amorphous regions [32].

A promising alternative to those techniques is glow-discharge optical emission spectroscopy (GD-OES), which allows for performing rapid depth profiling of selected elements through a plasma discharge burning between the sample surface, operated as cathode, and a counter electrode. In such a system, the plasma discharge continuously removes material from the sample surface by the impact of low-energy sputtering plasma ions while recording elemental optical emission from the removed species being present in an excited state in the discharge. Due to the low energy of the sputtering ions of $<100 \mathrm{eV}$ that is close to the sputtering threshold [33], alterations of the original sample surface due to the ion impact are lower than when using ion beam sputtering in XPS and AES with ion energies usually exceeding $1000 \mathrm{eV}$. The random inclination angle of the sputtering ions in GD-OES additionally helps to minimize potential alteration of the results due to the surface topography. Nevertheless, electric field enhancement effects may locally increase the sputter rate at sharp features of the surface topography. In contrast to XPS or AES, a surface analytical quantification of binding energies is not accessible by GD-OES.

Similar to XPS or AES, measurement and conversion of the acquired data to quantitative concentration versus depth profiles may be affected, e.g., by pronounced surface topography of the samples, different sputtering yield of the elements involved, or a non-planar ground of the sputtering crater as a result of plasma conditions. The depth resolution attained by GD-OES was estimated to be $\sim 15 \%$ of the actual sputtering depth, applying the specific method to equiatomic NiTi alloys [34]. Additionally, ignition and stabilization of the plasma at the beginning of each measurement require several milliseconds, corresponding to a few nanometers of the sample surface being removed before meaningful results can be attained. However, once a suitable method for a material system is optimized, measurements are quick and reproducible [35].

Taking benefit of its time and cost efficiency, in this work we apply GD-OES in order to analyze the depth extent of the laser-induced oxide layer generated upon femtosecond laser processing of three characteristic surface morphologies (LIPSS, Grooves, and Spikes) on Ti6Al4V titanium alloy and a polished (non-irradiated) sample as a reference. Our results for LIPSS are cross-checked with earlier depth-profiling AES results and complemented by gracing incidence $\mathrm{X}$-ray diffraction (XRD) measurements. 


\section{A brief review of oxidation effects in fs LIPSS formation}

Although fs-laser processing experiments are usually performed in air allowing oxidation reactions of the irradiated material with the ambient and reactive air environment, so far only little research has been devoted to chemical effects accompanying the formation of LIPSS [36-39]. Particularly for the highly reactive material titanium, such effects were already shown to be relevant for the processing of LIPSS [30, 40-43].

For simplicity, most of the experimental studies rely on EDX. The method provides sufficient spatial resolution to resolve the LIPSS, but its depth resolution is typically limited to a few micrometers. Moreover, it was shown that EDX can seriously suffer from topographic artifacts and must be applied with care [30]. Most other surface analytical techniques such as XPS or (micro-) Raman spectroscopy cannot spatially resolve individual LIPSS ridges and, thus, provide information averaged over an ensemble of topographic LIPSS maxima and minima. Yet, only AES in combination with a depth-profiling ion sputtering approach provided sufficient lateral and depth resolution to reveal the local chemistry of LIPSS for both the LSFL and HSFL [30].

Landis et al. [41] processed ablative LSFL on high-purity titanium (99.99\%) surfaces in different ambient atmospheres (air, oxygen, nitrogen) upon irradiation with Ti:sapphire fslaser pulses ( $100 \mathrm{fs}$ pulse duration, $0.25 \mathrm{~J} / \mathrm{cm}^{2}$ fluence, $1 \mathrm{kHz}$ pulse repetition rate). Micro-Raman spectroscopy revealed the formation of amorphous $\mathrm{TiO}_{2}$ (fully consistent with our results published in [30]), but given the large width of the Raman peaks, contributions of other amorphous titanium oxides are possible [41]. For the fs-laser treatment in oxidizing atmospheres (air, oxygen), the authors identified by XPS the formation of a surface layer consisting mainly of $\mathrm{TiO}_{2}$ with a possible admixture of less oxidized $\mathrm{Ti}_{2} \mathrm{O}_{3}$ or $\mathrm{TiO}$ [41].

Kirner et al. [30] performed spatially and depthresolved chemical analyses on HSFL and ablative LSFL on titanium and Ti6Al4V alloy by employing AES, XPS, EDX, and micro-Raman spectroscopy. A graded oxide layer with an extent $>150 \mathrm{~nm}$ was reported for the HSFL/ LSFL consisting mainly of amorphous $\mathrm{TiO}_{2}$ (near surface) and microcrystalline/amorphous $\mathrm{Ti}_{2} \mathrm{O}_{3}$ (subsurface) [30].

For another type of LSFL (so-called thermochemical LIPSS, TLIPSS, oriented parallel to the laser beam polarization) formed upon irradiation of thin titanium films by high repetition rate $(200 \mathrm{kHz})$ fs-laser pulses at fluences below the ablation threshold, micro-Raman spectroscopy identified $\mathrm{TiO}_{2}$ in the form of nanocrystalline rutile [43].

Peng et al. [44] studied the formation of Spikes on grade 2 titanium upon ultrafast laser scan processing
( 35 fs pulse duration, fluences between 0.4 and $3.5 \mathrm{~J} / \mathrm{cm}^{2}$, $1 \mathrm{kHz}$ pulse repetition rate) by using a dual-beam focused ion beam-scanning electron microscope to create cross sections for SEM, EDX, TEM, and selected area electron diffraction. The near-surface region of the irradiated material was found to contain a re-solidified porous layer of fine-grained $\alpha$-Ti. Cross-sectional EDX indicated near the surface the incorporation of up to 25 at.\% of oxygen in the fine-grained layer that may extend a few micrometers into depth.

\section{Experimental details}

Grade 5 titanium alloy (Ti6Al4V) was purchased from Schumacher Titan GmbH (Solingen, Germany) as a rod material of $25 \mathrm{~mm}$ diameter. The rods were cut into cylinders of $8 \mathrm{~mm}$ height. Subsequently, the top surfaces of the cylinders were mechanically polished, resulting in surface average and root-mean-squared roughness parameters $R_{\mathrm{a}}=5 \mathrm{~nm}$ and $R_{\mathrm{rms}}=6 \mathrm{~nm}$, respectively.

If exposed to air at room temperature and due to their high chemical reactivity, metallic titanium surfaces are typically covered by passivating native oxide layers, which consist mainly of amorphous and nanocrystalline $\mathrm{TiO}_{2}$. Additionally, these surfaces are covered by hydrocarbon molecules adsorbed from the ambient air. In our previous work, similar polished samples consisting of pure titanium $(99.6 \%)$ were characterized in detail by variable angle spectroscopic ellipsometry (VASE) [45] and by XPS [30]. The VASE measurements indicated the presence of a native $\mathrm{TiO}_{2}$ layer of $\sim 4 \mathrm{~nm}$ thickness [45]. XPS was used to determine the chemical composition at the polished surface, accounting to 14.5 at.\% of Ti, 48.5 at.\% of O, 31.8 at.\% of C, and 1.4 at.\% of N (rest 3.8 at.\%) [30]. Similar amounts of oxygen and carbon from contaminating hydrocarbons can be expected at the surface of the polished titanium alloy samples as well.

\subsection{Femtosecond laser processing and characterization of LSFL, Grooves, and Spikes}

The laser system used was a titanium:sapphire-amplified laser system (Compact Pro, Femtolasers) delivering pulses of $790 \mathrm{~nm}$ center wavelength and $30 \mathrm{fs}$ duration (FWHM) at a pulse repetition rate of $1 \mathrm{kHz}$. The pulses were focused using a spherical dielectric mirror of $500 \mathrm{~mm}$ focal length, obtaining a $1 / \mathrm{e}^{2}$ beam diameter of $2 w_{0} \sim 130 \mu \mathrm{m}$ at the focus. The positioning of the sample was done by three motorized linear translation stages, $X-Y$-axes for the positioning on the processing plane and the $Z$-axis to control the laser beam focus at the sample surface. In order to ensure perpendicularity of the surface with respect to the laser beam axis, 
the sample was locked in a holder that allowed for controlling the sample tilt. Since the laser is pulsed, the production of an area is the result of overlapping subsequent pulses to generate lines and overlapping lines to process areas, taking into account as controlling parameters the peak fluence $\left(\phi_{0}\right)$, the effective number of pulses per spot diameter $\left(N_{\text {eff_1D }}\right)$, and the line separation distance $(\Delta)$, following the expressions published in [46]. The irradiated areas were squares of $3.1 \times 3.1 \mathrm{~mm}^{2}$ or $7 \times 7 \mathrm{~mm}^{2}$ to allow enough space for the chemical and tribological characterizations in both directions, along and across the laser irradiation. During the laser irradiation, an air suction system was used to extract the generated gases and the ablation products.

\subsection{Surface characterization}

The sample surfaces were inspected by optical microscopy (Nikon, Eclipse L200) and characterized by scanning electron microscopy (Carl Zeiss, Gemini Supra 40). Selected surface morphologies were subjected to X-ray diffraction measurements for probing structural modifications at the laser-irradiated surface regions. The measurements (Seifert $\mathrm{GmbH}, \mathrm{XRD} 300 \mathrm{TT}$ ) were performed in a gracing incidence geometry (incident angle $\Omega=3.5^{\circ}$ ) using $\mathrm{Cu} \mathrm{K} \alpha$ radiation $(40 \mathrm{kV}, 40 \mathrm{~mA})$ with an X-ray beam collimated by a Göbel mirror. In this geometry, the probed area was about $6 \times 7$ $\mathrm{mm}^{2}$ (illumination width limited by a lead foil) adapted to the lateral dimension of the laser-processed region.

Quantitative depth profiles of Ti, Al, V, O, and C were determined by GD-OES (SPECTRUMA Analytik GmbH, GDA 750) using the GD-OES method as presented in [34]. Re-calibration and modification of the method were carried out using well-defined reference specimens to account for the additional elements $\mathrm{Al}$ and $\mathrm{V}$. The measurements were performed in radio frequency mode in order to allow for plasma ignition when working with samples covered by a non-conducting surface layer. Voltage and pressure were set to $700 \mathrm{~V}$ and $3 \mathrm{hPa}$, respectively. The measurements were pressure-regulated, and an anode with a diameter of $2.5 \mathrm{~mm}$ was used. Intensities of the respective elements were measured by photomultiplier tubes for Ti (399.864 nm), C (156.143 nm), Al (396.152 nm), V (411.179 nm), and O $(130.217 \mathrm{~nm})$. The intensity profiles were recorded as a function of the plasma discharge time and converted to quantitative mass concentration versus depth profiles on the basis of the comparative GD-OES method and a mean sputtering rate as determined after the measurements by evaluating the depth of the sputtering craters.

\subsection{Tribological tests}

Reciprocating sliding tribological tests (RSTTs) were performed with an in-house-built tribometer in ball-on-disk geometry [47]. The samples were tested in the regime of mixed friction with a stroke of $1 \mathrm{~mm}$ at a frequency of $1 \mathrm{~Hz}$ and with a normal force of $1 \mathrm{~N}$ against a hardened and polished ball made of steel as counterbody (100Cr6, diameter $10 \mathrm{~mm}$, average roughness $6 \mathrm{~nm}$ ) in a fully formulated, factory-fill engine oil (viscosity class $0 \mathrm{~W}-30$, Castrol 'VP-1'). At this normal force, the radius of the ball-sample contact area was estimated with a Hertzian deformation model as $\sim 36 \mu \mathrm{m}$. The corresponding elastic sample-ball deformation is $\sim 260 \mathrm{~nm}$ along with a maximum contact pressure of $\sim 360 \mathrm{MPa}$ [48]. As in previous experiments [49], the coefficient of friction (CoF) was measured during 1000 sliding cycles in two orthogonal directions, i.e., perpendicular and parallel to the laser-processed lines. Such a relatively low number of sliding cycles are suitable in most cases to characterize the tribological running-in phase. It allows to identify beneficial tribological effects [49] or the role of additives contained in the engine oil [48]. Note that our work does not address a specific tribological application. It is basic research and aims to provide new results by a systematic comparison of the tribological performance of fs-laser-generated LIPSS [49], Grooves, and Spikes under identical RSTT conditions. The experimental uncertainty range in the CoF measurements is \pm 0.02 . After the tribological tests, the samples were cleaned for five minutes in an ultrasonic bath with petroleum ether for removing residual lubricant. After the tribological tests, selected wear tracks were characterized via energy-dispersive X-ray spectroscopy (EDX, Thermo Fisher Scientific, Thermo NSS 3.1) at $5 \mathrm{kV}$

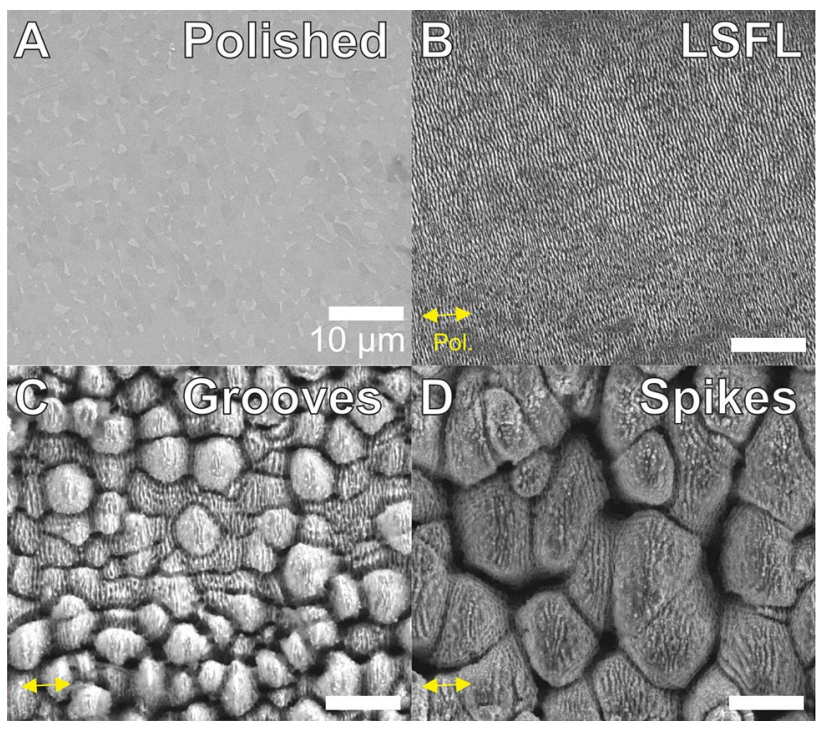

Fig. 1 Top-view scanning electron microscopy (SEM) images, of the titanium alloy surface (Ti6Al4V). a Micrograph of the polished sample surface, b LSFL, $\mathbf{c}$ Grooves and $\mathbf{d}$ for hierarchical Spikes. The yellow double arrow indicates the polarization direction of the laser beam 
acceleration voltage to identify the spatial elemental distribution on the surface.

\section{Results and Discussion}

Figure 1 contains scanning electron micrographs displaying the morphology of the different irradiated areas, including the free polished surface (Fig. 1a). Low-spatialfrequency LIPSS are displayed in Fig. 1b, where it is possible to resolve a pattern of parallel lines all perpendicular to the laser beam polarization indicated by a yellow arrow. In this case, the spatial period of the lines is between 500 and $700 \mathrm{~nm}$. These LSFL structures were produced using a pulse energy $E=30 \mu \mathrm{J}$, laser peak fluence $\phi_{0}=0.5 \mathrm{~J} / \mathrm{cm}^{2}$, a number of effective pulses per spot diameter of $N_{\text {eff_1D }}=40$, and a line separation distance of $\Delta=50 \mu \mathrm{m}$. When increasing both, the laser fluence and the effective number of pulses, different surface structures emerge, as the case of Grooves displayed in Fig. 1c $\left(E=151 \mu \mathrm{J}, \phi_{0}=2.5 \mathrm{~J} / \mathrm{cm}^{2}\right.$, $\left.N_{\text {eff_1D }}=100, \Delta=50 \mu \mathrm{m}\right)$ and Spikes in Fig. $1 \mathrm{~d}(E=181 \mu \mathrm{J}$, $\left.\phi_{0}=3.0 \mathrm{~J} / \mathrm{cm}^{2}, N_{\text {eff_1D }}=400, \Delta=50 \mu \mathrm{m}\right)$. These morphologies represent hierarchical structures going from protrusions in the micrometer regime to nanoripples spread all over the irradiated surface.

\subsection{XRD characterization}

In order to identify the chemical/structural composition of the oxide that is present at the surface of the structures, one useful technique is the X-ray diffraction (XRD). Since the

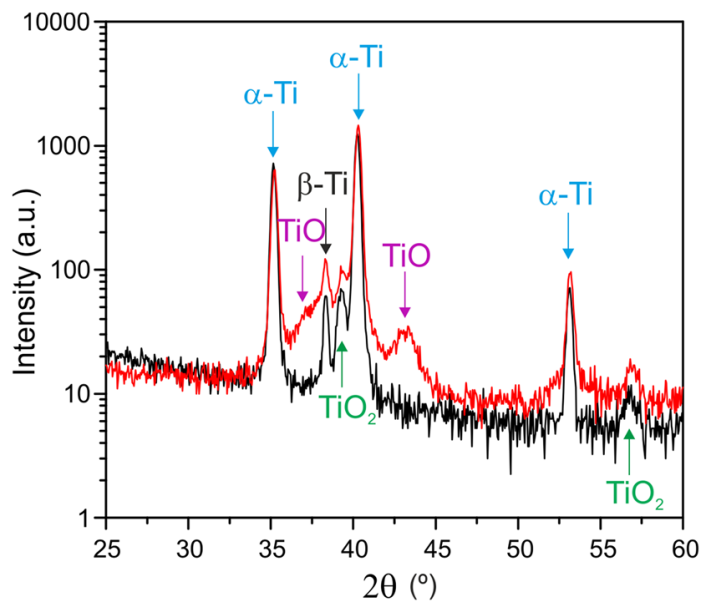

Fig. 2 X-ray diffraction (XRD) curves of the titanium alloy sample including the reference of a non-irradiated area (black line) and the corresponding measurement recorded at a laser-irradiated area with Spikes (red line). Peaks that correspond to metallic Ti ( $\alpha$ - and $\beta$-Ti phases), titanium oxide (TiO), and titanium dioxide $\left(\mathrm{TiO}_{2}\right)$ are indicated. Note the logarithmic intensity scale chemical composition of LSFL has been already studied via XPS [41] and AES [30] and was reported to be covered by $\mathrm{TiO}_{2}$, whereas the material structure was found to be amorphous by micro-Raman spectroscopy [30,41], in the present study we analyze the surface morphology generated with the highest fluence, i.e., in our case the Spike structures. Figure 2 shows the XRD measurements of the polished titanium alloy sample (non-irradiated, black line) as a reference and the one recorded in the irradiated area (red line) with Spike structures. The peaks labeled with $\alpha$ and $\beta$ (blue and black labels, respectively) indicate the corresponding metallic phases of titanium. Their peak width is similar for both the reference and the Spikes region, indicating that they both arise from the bulk material. For the case of the titanium oxide (TiO, violet label) and dioxide $\left(\mathrm{TiO}_{2}\right.$, green label), the peak width is increased, indicating the presence of polycrystalline oxides. An estimate of the widths of these peaks based on the Scherrer equation indicates maximum crystallite sizes of $\sim 30 \mathrm{~nm}$ in the oxide layer probed by XRD. This is reasonable here since significantly larger peak fluences and number of pulses were used for the Spikes processing when compared to the LSFL processing, where predominantly amorphous $\mathrm{TiO}_{2}$ was observed in a graded surface layer extending more than $200 \mathrm{~nm}$ into depth [30].

\subsection{GD-OES characterization of LSFL, Grooves, and Spikes}

Figure 3 compares two different representations of the depth-profile results obtained via GD-OES for the polished (non-irradiated) sample shown in Fig. 1a. The plotted results both correspond to the same measurement recorded over a total time of $1.5 \mathrm{~s}$. From the average sputter rate of $100 \mathrm{~nm}$ per second, an additional abscissa providing the sputtered depth is linked at the top. Details of the experimental conditions for this characterization are provided in Sect. 3.2. Figure $3 \mathrm{a}$ presents the measured intensity profiles versus sputtered time/depth of the elements Ti (violet), C (blue), $\mathrm{V}$ (red), $\mathrm{Al}$ (yellow), and $\mathrm{O}$ (green). The measured intensities at the specific wavelengths are the direct result of the emission of each element in the glow-discharge plasma at a given moment. The conversion of the measured intensities to quantitative concentrations for each element depends on numerous factors including sputtering rate factors or specific emission characteristics. Thus, the conversion of measured intensities to quantitative concentrations requires a calibration method as described in [34], and the progression of quantitative concentration profiles may differ significantly from the progression of the intensity profiles. As an example, in Fig. 3b the mass concentration depth profiles corresponding to the intensity data of Fig. $3 \mathrm{a}$ are presented. In the following, depth profiles after quantification will be shown. 

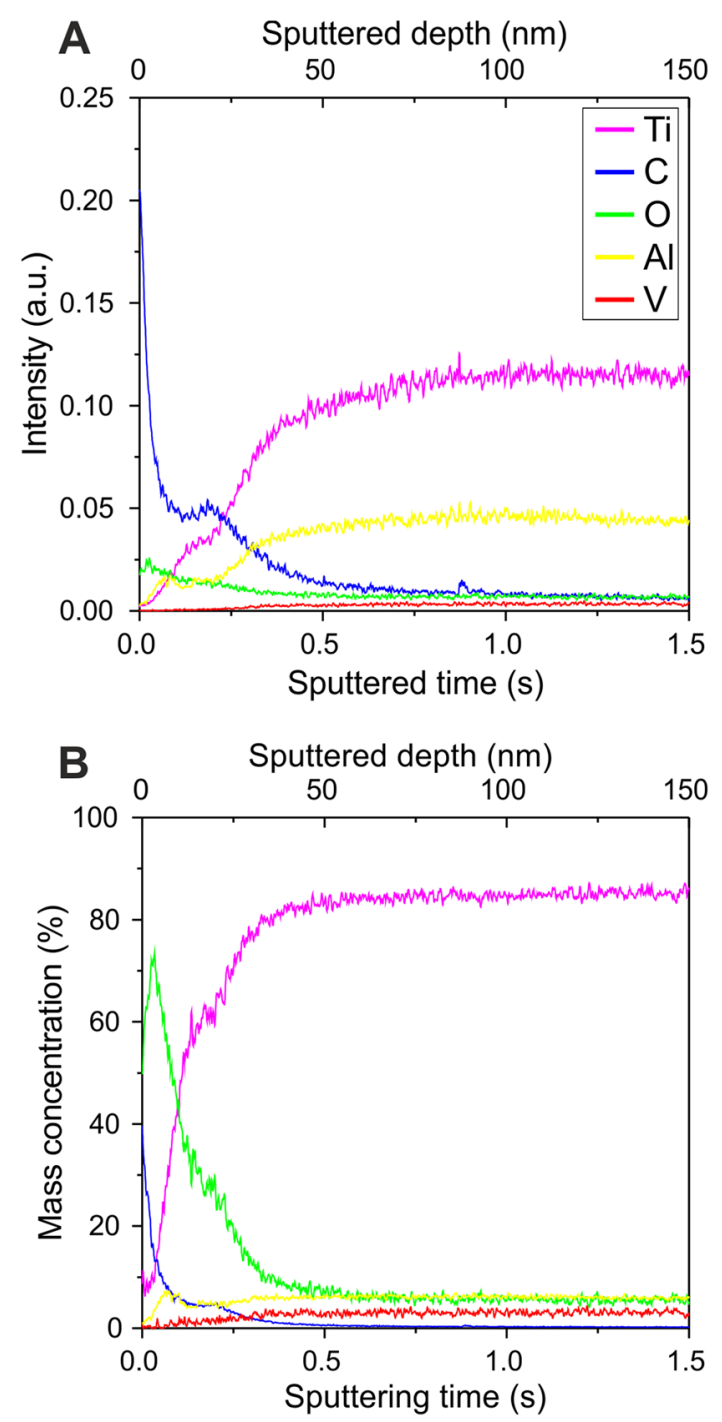

Fig. 3 GD-OES depth profiles of the same measurement at the polished (non-irradiated) Ti6Al4V sample in two different representations. a Emission intensity profiles versus sputtered time/depth, b derived mass concentration versus sputtered time/depth. The inset in (a) shows information of the displayed elements including Ti (violet), $\mathrm{C}$ (blue), O (green), Al (yellow), and V (red). The system sputters $100 \mathrm{~nm}$ of Ti6Al4V per second

Figure 4 provides the area plots of depth profiles on the polished surface (Fig. 4a, same data as in Fig. 3), for the LSFL (Fig. 4b), the Grooves (Fig. 4c), and the Spikes (Fig. 4d) under identical measuring conditions. The measurements were performed up to a sputtered depth of $\sim 2.5 \mu \mathrm{m}$. For easier intercomparison, the data are visualized for the top $500 \mathrm{~nm}$. At the polished surface (Fig. 4a), 10\% of $\mathrm{Ti}, \sim 35 \%$ of $\mathrm{C}$, and $\sim 50 \%$ of $\mathrm{O}$ are present with a low amount of $\mathrm{Al}$ and $\mathrm{V}$ (each around $2 \%$ ). High amounts of $\mathrm{O}$ and $\mathrm{C}$ at the very beginning of a measurement may partially be an artifact due to water vapor and organic contaminants adsorbed to the inner surface of the anode and the sample surface. The concentration of $\mathrm{C}$ at the sample surface $(\sim 35 \%)$ is consistent with the measurements of polished $\mathrm{Ti}$ samples characterized via XPS by Yasumaru et al. [42] and Kirner et al. [30], where carbon concentrations were $43.4 \%$ and $31.8 \%$, respectively. These notable carbon concentrations are attributed to the presence of hydrocarbon contaminants adsorbed at the surface. The effect strongly depends on the effective surface and is, therefore, observed after the laser irradiation [9] that is increasing the surface roughness/ porosity. Within a sputtered depth of $40 \mathrm{~nm}$, the signals of all elements approach a second regime, meeting the elemental composition of the Ti6Al4V alloy, i.e., $\sim 6 \% \mathrm{Al}, \sim 4 \% \mathrm{~V}$, and $\sim 0.2 \% \mathrm{O}$. The measurement of the oxide layer thickness is quantified here following the $50 \%$ criterion, defined as the distance from the surface when the $\mathrm{O}$ signal has decreased to half of the value detected at the surface. Applying this criterion, a native oxide layer thickness of $\sim 10 \mathrm{~nm}$ is obtained here for the polished sample surface. This value is consistent with the literature, which reports that the native oxide layer is spontaneously formed upon exposure to air and consists mainly of amorphous or nanocrystalline $\mathrm{TiO}_{2}$ (in line with the reference curve in our XRD measurements previously shown in Fig. 2), with typical thicknesses between 3 and $10 \mathrm{~nm}[50,51]$. The mild signal variation for depths $>40 \mathrm{~nm}$ likely arises from slowly altering plasma discharge conditions due to the increasing distance between sample surface and anode. Please note that the depth resolution attained by GD-OES in the present case is expected to be $\sim 15 \%$ of the actual sputtering depth, as detailed in the Introduction section.

At the LSFL-covered surface (Fig. 4b), the carbon signal extends to deeper positions inside the sputtered sample $(\sim 50 \mathrm{~nm})$ compared to the polished one shown in Fig. 4a. As it was mentioned before, this effect is related to the increase in the effective surface that allows more hydrocarbon contaminants to be adsorbed. The oxygen signal in this case extends more than $200 \mathrm{~nm}$ into depth, which is consistent with previous depth-profiling AES measurement [30]. Applying the above-mentioned 50\% criterion, an oxide layer thickness $\sim 40 \mathrm{~nm}$ is determined. In the region of this oxide layer, $\mathrm{V}$ is widely depleted. Interestingly, the $\mathrm{Al}$ concentration shows a near-surface minimum at $\sim 20 \mathrm{~nm}$ and a local maximum around $50 \mathrm{~nm}$ depth. Presumably, the high Gibbs free energy of formation of $\mathrm{Al}$ oxides leads to a preferred oxidation of $\mathrm{Al}$ already in the inner part of the oxide. Details of this effect remain to be assessed in future work. A diffusion of $\mathrm{Al}$ from the oxide toward the metal substrate is unlikely due to the ionic nature of the bonds. The arrow in Fig. $4 \mathrm{~b}$ marks a measurement artifact, which arises due to a temporary instability of the plasma discharge.

In the regions of laser-processed Grooves (Fig. 4c), carbon is detected few hundred of $\mathrm{nm}$ in depth due to a significant increase in the surface roughness. The $\mathrm{O}$ signal extends 
Fig. 4 Depth profiles obtained from the glow-discharge optical emission spectroscopy (GD-OES) showing the mass concentration of $\mathrm{Ti}$ (violet), $\mathrm{C}$ (blue), $\mathrm{O}$ (green), $\mathrm{Al}$ (yellow), and $\mathrm{V}$ (red) at different sputtered depths for a unirradiated flat polished sample, b LSFL, c Grooves, and d Spikes. The arrow in (b) marks a measurement artifact arising from a plasma instability

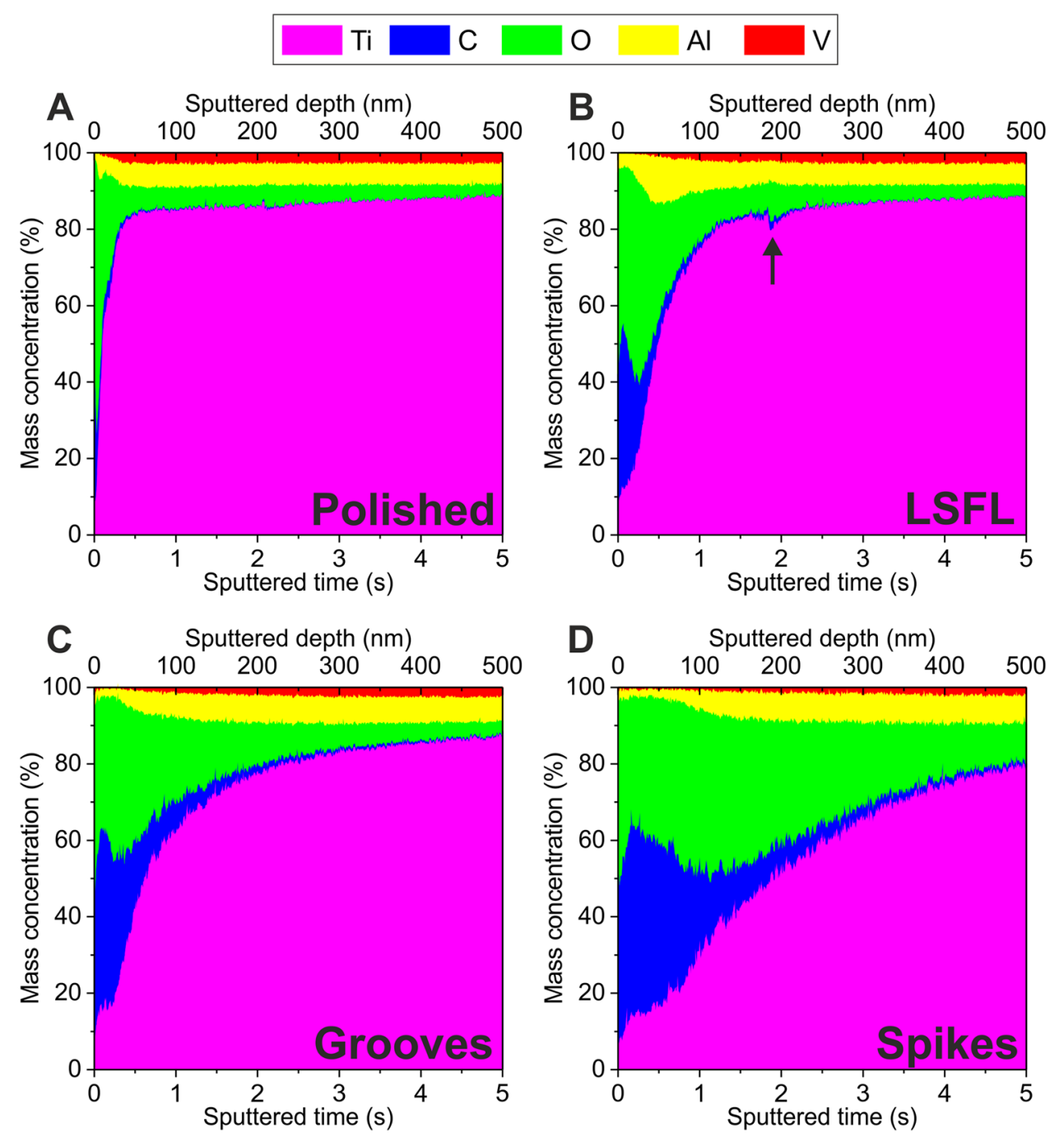

more than $300 \mathrm{~nm}$ into the bulk of the Ti alloy, resulting in an oxide layer thickness of $\sim 60 \mathrm{~nm}$ following the $50 \%$ criterion. The reduction of $\mathrm{Al}$ in the near-surface region is still evident.

For the Spikes surface morphology (Fig. 4d), the thickness of the laser-induced oxide layer exceeds $165 \mathrm{~nm}(50 \%$ criterion). Such thicknesses of the laser-induced graded oxide layer are consistent with the cross-sectional TEM/ EDX analyses of Peng et al. [44] reporting even larger thicknesses for somewhat different experimental conditions. Comparing the amount of $\mathrm{C}$ and $\mathrm{O}$ recorded for the LSFL (Fig. 4b), Grooves (Fig. 4c), and Spikes (Fig. 4d) shows that the presence of carbon is more confined to the surface than that of the $\mathrm{O}$. This may indicate the presence of a (nano-) porosity of the near-surface layer that is capable to adsorb hydrocarbons from the atmosphere and release them during the GD-OES measurements. Such a porosity of the nearsurface laser-modified layer was experimentally reported by Peng et al. for fs-laser-generated Spikes on Ti [44].
From a direct comparison of the spectra shown in Fig. 4, for the different surface morphologies, it clearly follows that the polished reference surface has the lowest concentration of carbon, while its concentration increases among the LIPSS, the Grooves, and the Spikes. Simultaneously, the surface roughness is the smallest for the polished surface and is increased for the laser-processed surfaces, being largest for the Spikes (compare Fig. 1a-d). Moreover, it is known that the increased $\mathrm{C}$ content after fs-, ps-, or ns-laser processing of metals arises from hydrocarbon contamination, as the laser-processed surface contains $\mathrm{OH}$ groups [9, 42, $52,53]$. Hence, it is very likely that the variation of the carbon concentration among the different surface morphologies arises here from the increased surface roughness and surface porosity, creating a larger effective surface area on which the hydrocarbon molecules are adsorbed. 
Fig. 5 Tribological performance of the samples after irradiation. a Sketch of the tribology setup using a steel ball of $100 \mathrm{Cr} 6$ on the surface of the Ti6Al4V alloy sample. The sketch shows the principle of the setup where a load of $1 \mathrm{~N}$ is applied on the ball to be in linear motion along $1 \mathrm{~mm}$ in the direction defined by the red arrow, with a frequency of $1 \mathrm{~Hz}$. The final wear track achieved after 1000 sliding cycles is shown in (b) for the free surface and in (c) for a Spike-covered area as optical micrographs. The scale bar is the same for both images. SEM micrographs of the wear track on the different areas are shown in (d) for the initially polished surface, e LSFL, f Grooves, and g Spikes
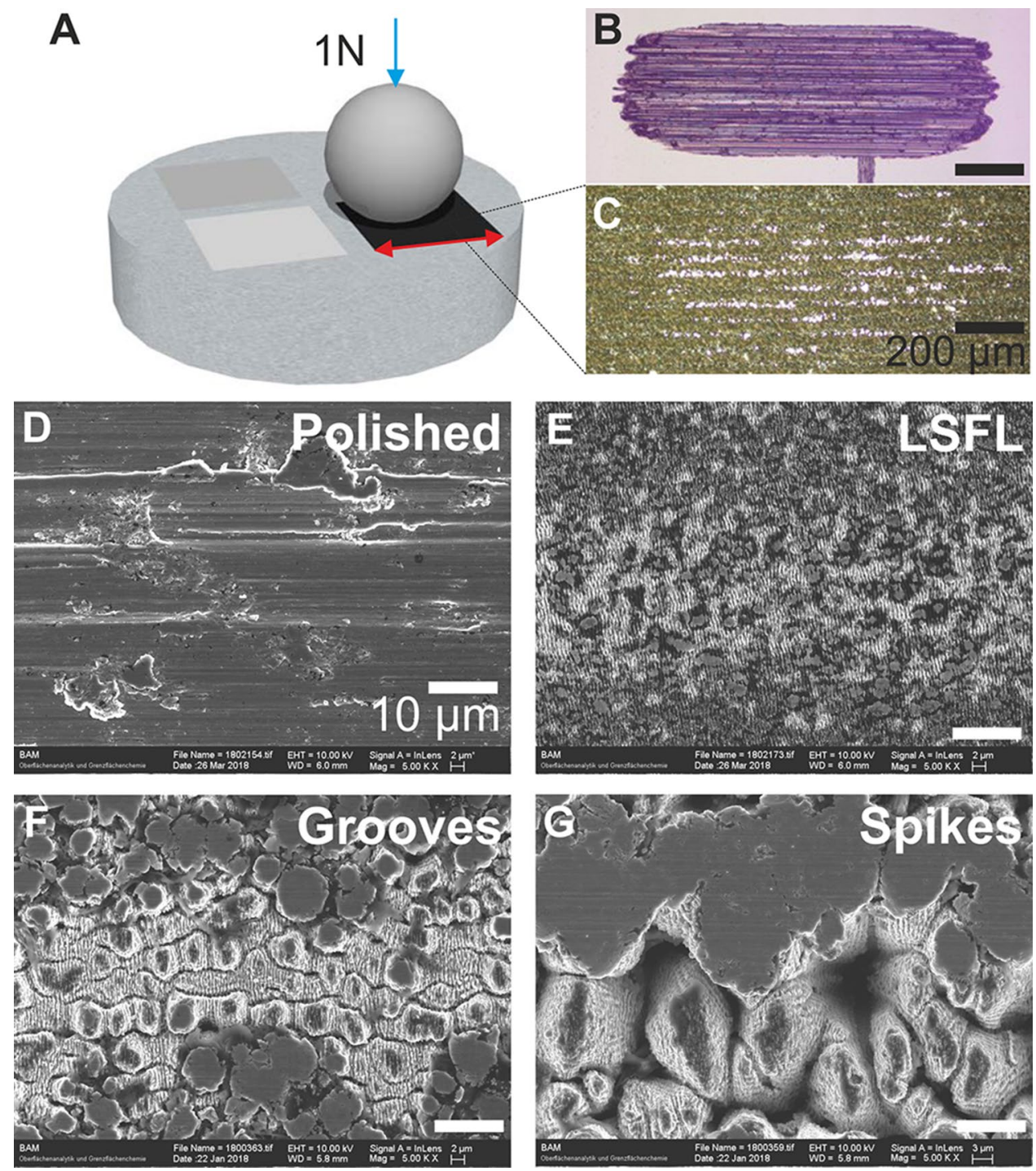

\subsection{Tribological performance of LSFL, Grooves, and Spikes}

Figures 5 and 6 present the results of the tribological characterization via RSTT of the three different laser-generated morphologies on titanium alloy, i.e., LSFL, Grooves, and Spikes and the polished flat surface. Figure 5 assembles a sketch of the RSTT geometry (Fig. 5a) along with top-view optical micrographs of the generated wear tracks on the polished Ti6Al4V alloy surface (Fig. 5b) and the Spike-covered surface (Fig. 5c). Additionally, top-view SEM micrographs revealing details from the wear tracks are presented (Fig. 5d: initially polished, Fig. 5e: LSFL, Fig. 5f: Grooves, Fig. 5g: Spikes). It is evident that on all laser-generated morphologies the topmost regions have been smoothened and partly worn during the RSTT, but the structures were not completely removed. The wear track and surface damage left at the polished surface is much larger than in the laser-processed regions.

Figure 6 assembles the corresponding measurements of the coefficient of friction versus the number of sliding cycles for all the laser-generated morphologies, i.e., LSFL (Fig. 6a), Grooves (Fig. 6b), and Spikes (Fig. 6c). To stay compatible with our previous works $[48,49,54]$, the $\mathrm{CoF}$ was measured at a normal load of $1 \mathrm{~N}$ during 1000 sliding cycles between the sample surface and the steel counterbody lubricated by the commercial engine oil. In all graphs, a curve obtained for the polished Ti6Al4V alloy surface is added as a reference (black line). The red and the blue curves represent measurements performed in the direction parallel (red line) or perpendicular (blue line) to the direction of the laser-processed lines. The mean value of the $\mathrm{CoF}$ as averaged over all cycles is indicated at the right for each curve. The $\mathrm{CoF}$ values for the initially polished surface vary between $\sim 0.37$ and $\sim 0.77$ with an average of 0.53 . Such high 

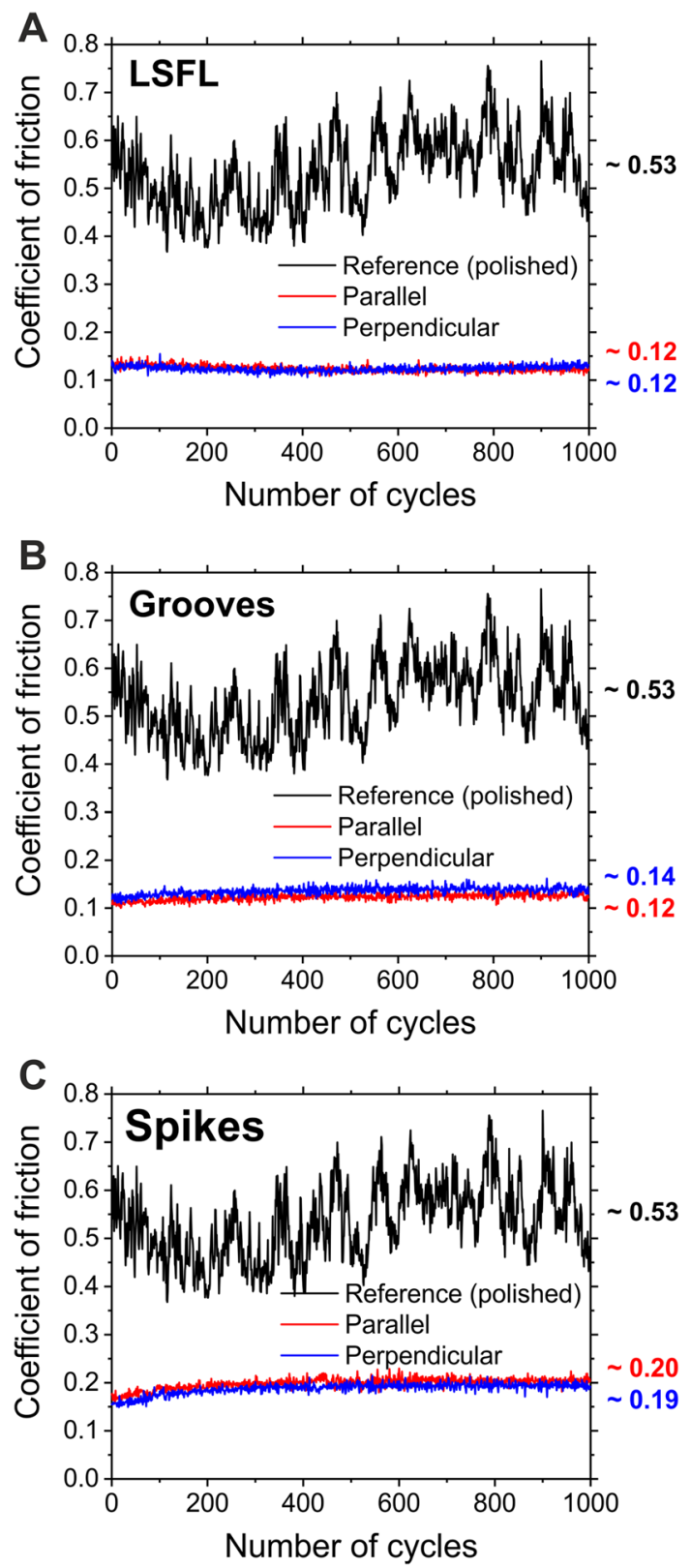

Fig. 6 Plots of the coefficient of friction versus the number of cycles of the tribological characterization using the setup sketched in Fig. 5a for a LSFL, b Grooves, and $\mathbf{c}$ Spikes on titanium alloy. All the areas were characterized using a fully formulated factory-fill engine oil (Castrol VP-1). The black line in all plots corresponds to a measurement performed at the polished surface (as reference), where the average value is 0.53 . The red and blue lines in each plot indicate the coefficient of friction recorded along and across the laser-processed ablation line structures, respectively

values indicate a severe damage of the polished surface and are consistent with the micrographs shown in Fig. 5b, d. These values are $~ 20-30 \%$ larger than previously reported in [49], which may arise from the severe surface damage generated and acting here during the RSTT.

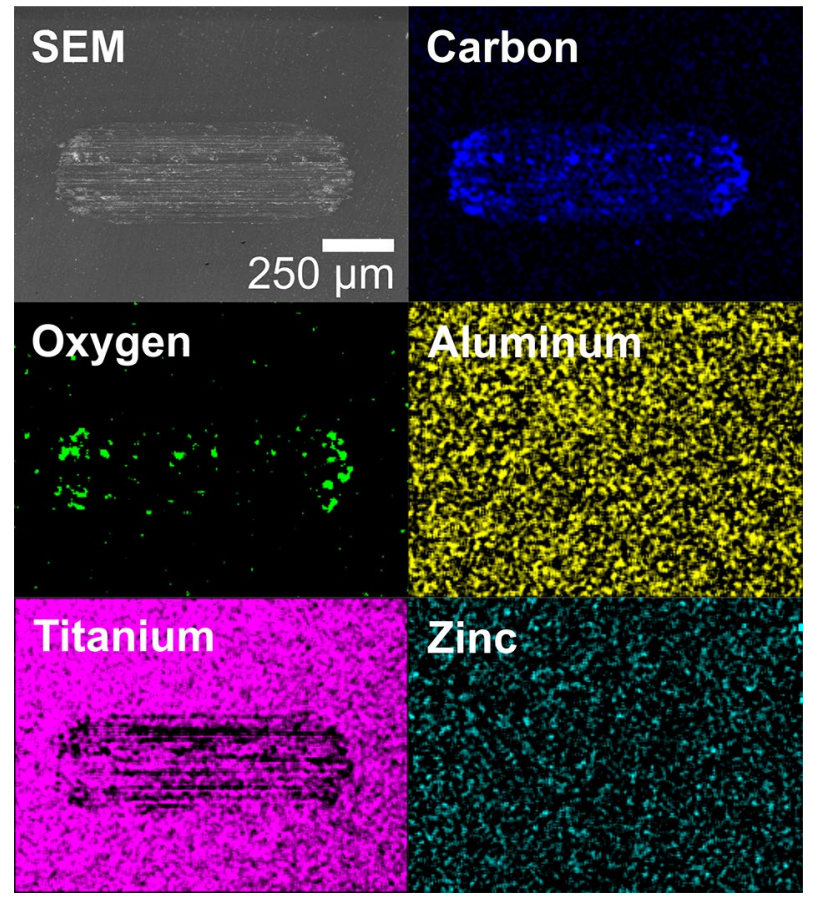

Fig. 7 EDX spatial distribution maps of the wear track generated on the polished titanium alloy samples. At the top left SEM micrograph, the wear track is clearly visible on the initially polished surface. The rest of the figures correspond spatially with the initial SEM micrograph displaying the spatial distribution of carbon $(\mathrm{C}-$ blue $)$, oxygen (O-green), aluminum (Al-yellow), titanium (Ti-magenta), and zinc ( $\mathrm{Zn}-$ cyan)

In contrast, the CoF measured in the LSFL-covered titanium alloy surface area (Fig. 6a) is reduced by a factor $4-5$ at an average level of $\sim 0.12$ in both sliding directions compared to the polished reference surface and shows smooth curves. These measurements confirm our previous results, where very similar CoFs and-within the experimental uncertainty-no significant influence on the sliding direction were reported [49]. The CoFs measured at the Grooves (Fig. 6b) indicate very similar results to that at the LSFL, i.e., mean $\mathrm{CoF}$ values of 0.12 and 0.14 in the direction parallel and perpendicular to the ablation lines, respectively. For the Spike-covered surface morphology (Fig. 6c), the CoF starts around $\sim 0.15$ and then slowly increases to a saturation value around $\sim 0.2$. In view of the results presented in Figs. 5 and 6, the LSFL morphologies feature the best tribological performance among the three different laser-generated surface morphologies. Hence, it was selected for a more detailed chemical characterization of the wear track.

For comparison, a wear track generated at the polished and at the LSFL-covered Ti6Al4V alloy surface was selected and characterized through EDX. The corresponding maps of the spatial distribution of the elements arising from the sample material $(\mathrm{Ti}, \mathrm{Al})$, the lubricant engine oil $(\mathrm{C}, \mathrm{O}, \mathrm{Zn})$, 


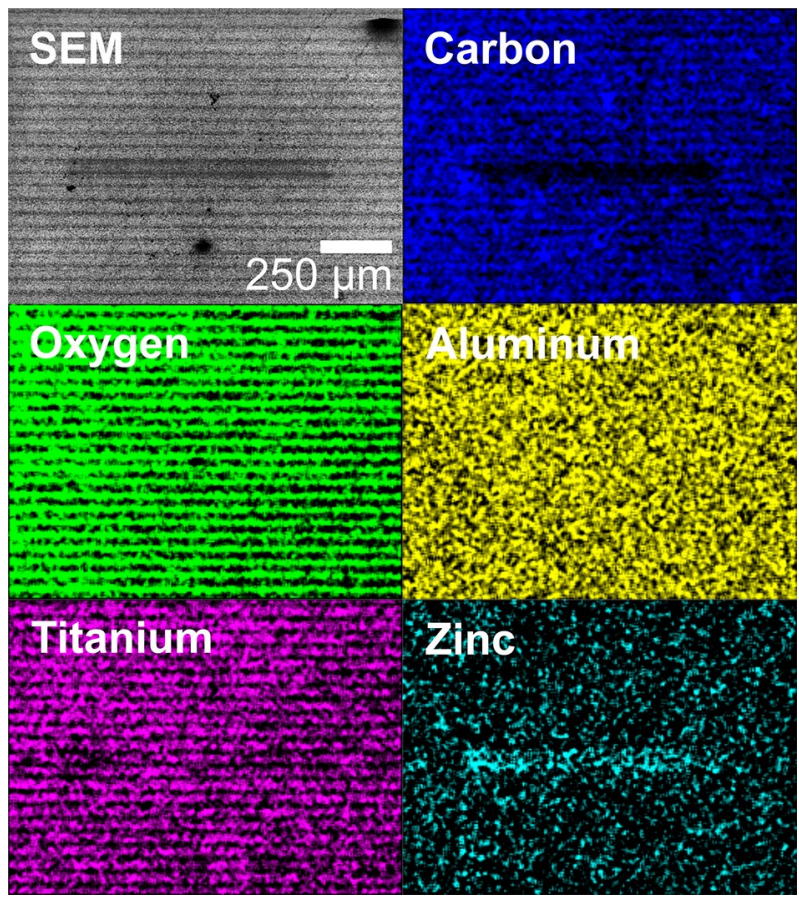

Fig. 8 EDX spatial distribution maps of the wear track generated on the LSFL-covered laser-irradiated area. At the top left, the corresponding SEM micrograph is provided. The rest of the figures correspond spatially with the initial SEM micrograph displaying the spatial distribution of carbon $(\mathrm{C}-$ blue $)$, oxygen $(\mathrm{O}$ - green), aluminum (Al-yellow), titanium (Ti-magenta), and zinc ( $\mathrm{Zn}-\mathrm{cyan})$. The tribological test was performed along $1 \mathrm{~mm}$ on the horizontal axis of the images shown

and environmental reactions $(\mathrm{O}, \mathrm{C})$ are assembled in Figs. 7 and 8 , respectively.

The wear track generated on the polished surface is clearly visible in the SEM micrograph of Fig. 7. It exhibits an increased $\mathrm{C}$ and $\mathrm{O}$ content at both of its ends, indicating residuals of the lubricant being still present at the surface. The native oxide on the Ti6Al4V alloy surface layer could not be detected by EDX at the chosen conditions as its thickness of $\sim 10 \mathrm{~nm}$ is more than one order of magnitude smaller than the EDX information depth (usually in the few micrometer range). $\mathrm{Al}$ contained in the alloy and $\mathrm{Zn}$ contained in the wear-reducing additives of the engine oil are uniformly distributed across the elemental maps at low signal levels close to the noise limit. In contrast, the signal of titanium is reduced within the wear track at the locations covered by $\mathrm{C}, \mathrm{O}$ and potentially from material transferred from the tribological counterbody during the RSTT.

The wear track generated at the LSFL-covered surface is less visible in the SEM micrograph of Fig. 8. The maps of $\mathrm{O}$ and $\mathrm{Ti}$ both show pronounced horizontal line structures arising from the line-wise laser processing. At regions of increased laser-induced oxidation, the corresponding Ti signal is reduced, and vice versa. In the maps of both elements $(\mathrm{Ti}, \mathrm{O})$, the wear track is hardly visible indicating that (i) the laser-induced oxide layer remained intact and (ii) tribo-oxidation effects during the RSTT are less effective than the laser-induced oxidation effects. Within the wear track, the signal of carbon is reduced, while the signal of $\mathrm{Zn}$ is increased. The latter observations are in line with the previous results obtained for tribological tests on LSFL-covered steel surfaces under similar test conditions [54]. The increased signal of $\mathrm{Zn}$ indicates the formation of a thin superficial tribo-layer from the anti-wear additive ZDDP (zinc dialkyl dithiophosphate) contained in the engine oil $[48,54]$.

\section{Conclusions}

Laser-induced oxide layers produced on LSFL, Grooves, and Spikes when irradiating Ti6Al4V alloy with femtosecond laser pulses have been quantified by depth profiling via glow-discharge optical emission spectroscopy. The approach allows for identifying thickness ranges of the oxide layers and for determining the elemental distribution of Ti, Al, V, and $\mathrm{C}$ in high detail due to the large area of measurement, the continuous evaluation of emissions during the discharge and sputtering, and the high sputtering rates. Complementary information on the chemical composition on specific areas has been obtained from gracing incidence XRD to identify the types of oxide formed. EDX characterization of samples used for friction coefficient measurements visualized the maps of the spatial distribution of the main elements present at the surface after the reciprocating sliding tribological tests, demonstrating the presence of oxygen along the laser-irradiated lines. Even for the weakest laser treatment generating the LSFL, the laser-induced oxide layer of $\sim 40 \mathrm{~nm}$ thickness (50\% criterion) stayed widely intact during our tribological tests. These oxides along with the nanoscale topographical alterations are ultimately responsible for the friction coefficient reduction measured on the laser-structured areas.

Acknowledgements Open Access funding provided by Projekt DEAL. The authors would like to thank S. Binkowski (BAM 6.3) for polishing the samples, S. Benemann (BAM 6.1) for the SEM and EDX characterizations, M. Sahre and T. Lange (both BAM 6.7) for the XRD analyses, and N. Slachciak (BAM 6.3) for the support with the tribological tests. This work was supported through the European Horizon 2020 FETOPEN program by the projects "LiNaBioFluid" (Grant Agreement No. 665337) and "CellFreeImplant" (Grant Agreement No. 800832) and through the Horizon 2020 INDUSTRIAL LEADERSHIP program by the project "i-TRIBOMAT" (Grant Agreement No. 814494). Financial support by the German Research Foundation (DFG, UN 341/3-1) is gratefully acknowledged.

Open Access This article is licensed under a Creative Commons Attribution 4.0 International License, which permits use, sharing, 
adaptation, distribution and reproduction in any medium or format, as long as you give appropriate credit to the original author(s) and the source, provide a link to the Creative Commons licence, and indicate if changes were made. The images or other third party material in this article are included in the article's Creative Commons licence, unless indicated otherwise in a credit line to the material. If material is not included in the article's Creative Commons licence and your intended use is not permitted by statutory regulation or exceeds the permitted use, you will need to obtain permission directly from the copyright holder. To view a copy of this licence, visit http://creativecommons .org/licenses/by/4.0/.

\section{References}

1. J.E. Sipe, J.F. Young, J.S. Preston, H.M. van Driel, Phys. Rev. B 27, $1141(1983)$

2. S. Gräf, C. Kunz, S. Engel, T.J.Y. Derrien, F. Müller, Materials (Basel) 11, 1340 (2018)

3. J. Bonse, A. Rosenfeld, J. Krüger, J. Appl. Phys. 106, 104910 (2009)

4. F.R. Weber, C. Kunz, S. Gräf, M. Rettenmayr, F.A. Müller, Langmuir 35, 14990 (2019)

5. J. Bonse, S. Höhm, S.V. Kirner, A. Rosenfeld, J. Krüger, IEEE J. Sel. Top. Quantum Electron. 23, 9000615 (2017)

6. A.Y. Vorobyev, C. Guo, Laser Photonics Rev. 7, 385 (2013)

7. E. Stratakis, A. Ranella, C. Fotakis, Biomicrofluidics 5, 013411 (2011)

8. J. Reif, Chapter 2 in Advances in the Application of Lasers in Materials Science (ed. by P.M. Ossi), Springer Series in Materials Science 274, (Springer, Berlin, 2018)

9. A.-M. Kietzig, S.G. Hatzikiriakos, P. Englezos, Langmuir 25, 4821 (2009)

10. C. Florian, S.V. Kirner, J. Krüger, J. Bonse, J. Laser Appl. (2020, in press)

11. I. Gnilitskyi, T.J.Y. Derrien, Y. Levy, N.M. Bulgakova, T. Mocek, L. Orazi, Sci. Rep. 7, 8485 (2017)

12. Y. Fuentes-Edfuf, J.A. Sánchez-Gil, C. Florian, V. Giannini, J. Solis, J. Siegel, ACS Omega 4, 6939 (2019)

13. A. Aguilar, C. Mauclair, N. Faure, J.-P. Colombier, R. Stoian, Sci. Rep. 7, 16509 (2017)

14. A. Rudenko, C. Mauclair, F. Garrelie, R. Stoian, J.P. Colombier, Appl. Surf. Sci. 470, 228 (2019)

15. S. Höhm, M. Herzlieb, A. Rosenfeld, J. Krüger, J. Bonse, Appl. Surf. Sci. 374, 331 (2016)

16. J. Bonse, S. Baudach, J. Krüger, W. Kautek, M. Lenzner, Appl. Phys. A 74, 19 (2002)

17. S. Gräf, F.A. Müller, Appl. Surf. Sci. 331, 150 (2015)

18. J. Reif, C. Martens, S. Uhlig, M. Ratzke, O. Varlamova, S. Valette, S. Benayoun, Appl. Surf. Sci. 336, 249 (2015)

19. P. Gregorčič, M. Sedlacek, B. Podgornik, J. Reif, Appl. Surf. Sci. 387, 698 (2016)

20. G. Miyaji, K. Miyazaki, K. Zhang, T. Yoshifuji, J. Fujita, Opt. Express 20, 14848 (2012)

21. F. Gesuele, J.J.J. Nivas, R. Fittipaldi, C. Altucci, R. Bruzzese, P. Maddalena, S. Amoruso, Appl. Phys. A 124, 204 (2018)

22. R. Kuladeep, M.H. Dar, K.L.N. Deepak, D.N. Rao, J. Appl. Phys. 116, 113107 (2014)

23. T.J.Y. Derrien, R. Koter, J. Krüger, S. Höhm, A. Rosenfeld, J. Bonse, J. Appl. Phys. 116, 074902 (2014)

24. C.A. Zuhlke, T.P. Anderson, D.R. Alexander, Opt. Express 21, $8460(2013)$

25. K. Ahmmed, C. Grambow, A.-M. Kietzig, Micromachines 5, 1219 (2014)
26. S.V. Kirner, U. Hermens, A. Mimidis, E. Skoulas, C. Florian, F. Hischen, C. Plamadeala, W. Baumgartner, K. Winands, H. Mescheder, J. Krüger, J. Solis, J. Siegel, E. Stratakis, J. Bonse, Appl. Phys. A 123, 754 (2017)

27. C. Florian Baron, A. Mimidis, D. Puerto, E. Skoulas, E. Stratakis, J. Solis, J. Siegel, Beilstein J. Nanotechnol. 9, 2802 (2018)

28. A.-M. Kietzig, M. Negar Mirvakili, S. Kamal, P. Englezos, S.G.G. Hatzikiriakos, J. Adhes. Sci. Technol. 25, 2789 (2011)

29. J. Heitz, C. Plamadeala, M. Muck, O. Armbruster, W. Baumgartner, A. Weth, C. Steinwender, H. Blessberger, J. Kellermair, S.V. Kirner, J. Krüger, J. Bonse, A.S. Guntner, A.W. Hassel, Appl. Phys. A 123, 734 (2017)

30. S.V. Kirner, T. Wirth, H. Sturm, J. Krüger, J. Bonse, J. Appl. Phys. 122, 104901 (2017)

31. M. Može, M. Zupančič, M. Hočevar, I. Golobič, P. Gregorčič, Appl. Surf. Sci. 490, 220 (2019)

32. K.E. Freiberg, R. Hanke, M. Rettenmayr, A. Undisz, Pract. Metallogr. 53, 193 (2016)

33. R.K. Marcus, J.A.C. Broekaert, Glow Discharge Plasmas in Analytical Spectroscopy (Wiley, Chichester, 2003)

34. A. Undisz, R. Hanke, K.E. Freiberg, V. Hoffmann, M. Rettenmayr, Acta Biomater. 10, 4919 (2014)

35. S. Zende, K.E. Freiberg, F. Dorner, N.-A. Feth, A. Undisz, Shap. Mem. Superelasticity 5, 346 (2019)

36. J. Bonse, H. Sturm, D. Schmidt, W. Kautek, Appl. Phys. A 71, 657 (2000)

37. B. Öktem, I. Pavlov, S. Ilday, H. Kalaycıŏglu, A. Rybak, S. Yavaş, M. Erdoğan, F.Ö. Ilday, Nat. Photonics 7, 897 (2013)

38. A. Cunha, O.F. Zouani, L. Plawinski, A.M. Botelho do Rego, A. Almeida, R. Vilar, M.-C. Durrieu, Nanomedicine 10, 725 (2015)

39. X.-F. Li, C.-Y. Zhang, H. Li, Q.-F. Dai, S. Lan, S.-L. Tie, Opt. Express 22, 28086 (2014)

40. C. Zwahr, A. Welle, T. Weingärtner, C. Heinemann, B. Kruppke, N. Gulow, M. Große Holthaus, A.F. Lasagni, Adv. Eng. Mater. 21, 1900639 (2019)

41. E.C. Landis, K.C. Phillips, E. Mazur, C.M. Friend, J. Appl. Phys. 112, 063108 (2012)

42. N. Yasumaru, E. Sentoku, J. Kiuchi, Appl. Surf. Sci. 405, 267 (2017)

43. A.V. Dostovalov, V.P. Korolkov, S.A. Babin, Appl. Phys. B 123, 30 (2017)

44. E. Peng, R. Bell, C.A. Zuhlke, M. Wang, D.R. Alexander, G. Gogos, J.E. Shield, J. Appl. Phys. 122, 133108 (2017)

45. S.V. Kirner, N. Slachciak, A.M. Elert, M. Griepentrog, D. Fischer, A. Hertwig, M. Sahre, I. Dörfel, H. Sturm, S. Pentzien, R. Koter, D. Spaltmann, J. Krüger, J. Bonse, Appl. Phys. A 124, 326 (2018)

46. J. Bonse, G. Mann, J. Krüger, M. Marcinkowski, M. Eberstein, Thin Solid Films 542, 420 (2013)

47. D. Klaffke, M. Hartelt, Tribol. Lett. 1, 265 (1995)

48. J.J. Ayerdi, N. Slachciak, I. Llavori, A. Zabala, A. Aginagalde, J. Bonse, D. Spaltmann, Lubricants 7, 79 (2019)

49. J. Bonse, R. Koter, M. Hartelt, D. Spaltmann, S. Pentzien, S. Höhm, A. Rosenfeld, J. Krüger, Appl. Phys. A 117, 103 (2014)

50. X. Liu, P. Chu, C. Ding, Mater. Sci. Eng. R Rep. 47, 49 (2004)

51. E. Gemelli, N.H.A. Camargo, Matéria (Rio Janeiro) 12, 525 (2007)

52. J. Long, M. Zhong, H. Zhang, P. Fan, J. Colloid Interface Sci. 441, 1 (2015)

53. Z. Yang, X. Liu, Y. Tian, J. Colloid Interface Sci. 553, 268 (2019)

54. J. Bonse, S.V. Kirner, M. Griepentrog, D. Spaltmann, J. Krüger, Materials (Basel) 11, 801 (2018)

Publisher's Note Springer Nature remains neutral with regard to jurisdictional claims in published maps and institutional affiliations. 